\title{
Safety of CMR in patients with cardiac implanted electronic devices
}

\author{
El-Sayed H Ibrahim ${ }^{1 *}$, Laura Horwood ${ }^{1}$, Jadranka Stojanovska', Anil Attili', Luba Frank², Hakan Oral', Frank Bogun ${ }^{1}$ \\ From 19th Annual SCMR Scientific Sessions \\ Los Angeles, CA, USA. 27-30 January 2016
}

\section{Background}

CMR has been reported to be safe in patients with cardiac implantable electronic device (CIED), provided a specific protocol is followed. The goal of this study is to examine whether this is true for patients excluded from published protocols, e.g. CIED patients with abandoned leads or pacemaker dependency.

\section{Methods}

The protocol followed at our institution for MR imaging of CIED patients is as follows: 1) Necessity and absence of an alternative imaging modality; 2) Device-related relative contraindications, including the presence of abandoned leads, pacemaker dependency, and time to lead implant < 6 weeks, with the possibility of the ordering physician to overrule these exclusions. 3) A provider with CIED management expertise assesses the baseline device information, where the device was programmed according to the patient's needs with tachyarrhythmia detection and therapy disabled during the scan. 4) Device reinterrogation and reprogramming after completion of the scan, as well as at 1 week and 3 months after imaging.

\section{Results}

A total of 162 MR scans were obtained in 142 consecutive patients with CIED's (106 patients had defibrillators and 36 had pacemakers, as shown in Figure 1). 29 patients were pacemaker dependent and 11 patients had abandoned leads. Cardiac MR scans were performed in 94 patients (late gadolinium enhancement (LGE) was used to determine myocardial scar prior to ablation) and spinal/ brain scans were performed in 47 patients. In the cardiac scans, the images were non-diagnostic only in 4 patients due to extensive artifact from the implanted cardiac defibrillator (ICD). In 65 patients, LGE was detected without

${ }^{1}$ University of Michigan, Ann Arbor, MI, USA

Full list of author information is available at the end of the article

\begin{tabular}{|ll|}
\hline \multicolumn{1}{|c}{ Characteristics } & \multicolumn{1}{c}{ Patients } \\
\hline Total patient (n) & 142 \\
Gender (m/f) & $117 / 25$ \\
Age (years) & $63 \pm 12$ \\
Implanted device; type (n) & 142 \\
- ICD & 106 \\
- Pacemaker & 36 \\
Implanted device; manufacturer (n) & 142 \\
- Medtronic & 60 \\
- Boston Scientific & 35 \\
- Biotronic & 12 \\
- St Jude & 35 \\
Pacemaker (PM) dependent & 29 \\
- PM patients & 10 \\
- ICD patients & 19 \\
Abandoned leads (n) & 13 in 11 patients \\
- Atrial leads & 1 \\
- Ventricular pacing lead & 1 \\
- ICD lead dual coil & 4 \\
- CRT lead in SVC & 2 \\
- Epicardial pacing leads & 4 \\
- SVC coil & 1 \\
Implantation < 8 weeks & 1 (41 days post implant) \\
Cardiac MRI & 94 \\
Non-Cardiac MRI (n) & 47 \\
Device at ERI prior to MRI & 1 \\
Device at EOL prior to MRI & 1 \\
Total scans performed (n) & 162 \\
- single scan & 140 \\
- two scans & 12 \\
- > two scans & 8 \\
\hline Abbreviations: PM= pacemaker, ICD= implantable cardioverter \\
defibrillator, CRT= cardiac resynchronization therapy, SVC= \\
superior vena cava, ERI = battery: elective replacement indicator, \\
EOL= battery: end of life. & \\
Figure 1 Patients' characteristics. & \\
\hline & \\
\hline
\end{tabular}

artifact. No LGE was identified in 25 scans. Only one patient developed ventricular tachycardia (VT) during a spine scan and was removed from the scanner for device reactivation, which terminated VT without consequences. 


\begin{tabular}{|c|c|c|c|c|c|}
\hline Parameters & Pre MRI & Post MRI & $\begin{array}{c}1 \text { week post } \\
\text { MRI }\end{array}$ & $\begin{array}{c}3 \text { Mo post } \\
\text { MRI }\end{array}$ & $\begin{array}{c}\text { p-value } \\
\text { (pre/post) }\end{array}$ \\
\hline \multicolumn{6}{|l|}{ Battery } \\
\hline$-m V, n=82$ & $2.96 \pm 0.31$ & $2.94 \pm 0.32$ & $2.92 \pm 0.41$ & $2.91 \pm 0.34$ & ns \\
\hline$-\%, n=17$ & $86 \pm 27$ & $85 \pm 13$ & $78 \pm 13 *$ & $73 \pm 15^{*}$ & $<0.05$ \\
\hline - years, $n=43$ & $8.5 \pm 2.7$ & $8.3 \pm 2.6$ & $8.1 \pm 2.7^{*}$ & $8.1 \pm 2.8$ & $<0.05$ \\
\hline P-wave amplitude (mV) & $3.38 \pm 2.1$ & $3.43 \pm 2.1$ & $3.1 \pm 1.83$ & $3.47 \pm 2.2$ & $\mathrm{~ns}$ \\
\hline R-wave amplitude (mV) & $12.92 \pm 9.7$ & $12.9 \pm 9.8$ & $11.38 \pm 5.44$ & $11.55 \pm 5.26$ & ns \\
\hline R-wave amplit. LV lead(mV) & $19.94 \pm 5.5$ & $19.1 \pm 6.96$ & $20.87 \pm 0.52$ & $13.17 \pm 7.55$ & ns \\
\hline Atrial lead impedance $(\Omega)$ & $477 \pm 159$ & $474 \pm 159$ & $480 \pm 183 *$ & $479 \pm 127$ & $<0.05$ \\
\hline Ventricular lead imped. $(\Omega)$ & $492 \pm 143$ & $490 \pm 143$ & $471 \pm 141$ & $485 \pm 146$ & ns \\
\hline LV lead impedance $(\Omega)$ & $708 \pm 338$ & $703 \pm 339$ & $712 \pm 327^{*}$ & $671 \pm 307$ & $<0.05$ \\
\hline Shock lead impedance $(\Omega)$ & $57 \pm 17$ & $58 \pm 17$ & $54 \pm 14 *$ & $57 \pm 18$ & $<0.05$ \\
\hline SVC shock impedance $(\Omega)$ & $55 \pm 9$ & $54 \pm 9$ & $54 \pm 9$ & $56 \pm 10$ & ns \\
\hline Atrial lead capture (V) & $0.79 \pm 0.48$ & $0.79 \pm 0.5$ & $0.75 \pm 0.31$ & $0.71 \pm 0.33$ & ns \\
\hline RV lead capture $(\mathrm{V})$ & $0.89 \pm 0.32$ & $0.92 \pm 0.33$ & $0.91 \pm 0.41$ & $0.92 \pm 0.36$ & ns \\
\hline LV lead capture (V) & $1.48 \pm 0.8$ & $1.55 \pm 0.85$ & $1.48 \pm 0.77$ & $1.52 \pm 0.76$ & ns \\
\hline
\end{tabular}

No other adverse events were noted. The device parameters essentially remained the same immediately, 1 week, and 3 months after the scans (Figure 2).

\section{Conclusions}

In the analyzed cohort of 142 patients, only in one pacemaker dependent patient, the heart rate dropped from 90 to $50 \mathrm{bpm}$ during spinal imaging using a fast spin echo sequence (specific absorption rate $(\mathrm{SAR})=1.89 \mathrm{~W} / \mathrm{Kg}$ ) due to elevated noise rate of the ICD. In this patient, a prior cardiac scan was performed a week earlier where no problem was observed, as the cardiac LGE sequences have low SAR of $0.11 \mathrm{~W} / \mathrm{Kg}$. Therefore, with the protocol described in this study, CMR imaging can be safely performed in CIED patients without exposing the patients to risk, despite pacemaker dependency, presence of abandoned leads, and other CIED contraindications. This study recommends that CMR imaging be available to more CIED patients who can benefit from it.

\section{Authors' details}

${ }^{1}$ University of Michigan, Ann Arbor, MI, USA. ${ }^{2}$ University of Texas Medical Branch, Galveston, TX, USA.

Published: 27 January 2016
doi:10.1186/1532-429X-18-S1-0123

Cite this article as: Ibrahim et al:: Safety of CMR in patients with cardiac implanted electronic devices. Journal of Cardiovascular Magnetic Resonance 2016 18(Suppl 1):0123.
Submit your next manuscript to BioMed Central and take full advantage of:

- Convenient online submission

- Thorough peer review

- No space constraints or color figure charges

- Immediate publication on acceptance

- Inclusion in PubMed, CAS, Scopus and Google Scholar

- Research which is freely available for redistribution 